\title{
Sobre el estatus taxonómico de Liolaemus islugensis Ortiz y Marquet, 1987 (Squamata: Liolaemidae)
}

\section{On the taxonomic status of Liolaemus islugensis Ortiz and Marquet, 1987 (Squamata: Liolaemidae)}

\section{Margarita Ruiz De Gamboa ${ }^{1,2,3^{*}}$ \& Juan Carlos Ortiz²}

${ }^{1}$ Centro de Investigación en Medio Ambiente (CENIMA), Universidad Arturo Prat, Casilla 121, Iquique, Chile.

'Laboratorio de Sistemática y Conservación de Herpetozoos (SyCo-Lab), Departamento de Zoología, Facultad de Ciencias Naturales y Oceanográficas, Universidad de Concepción, Concepción, Chile.

${ }^{3}$ Programa de Doctorado en Sistemática y Biodiversidad, Departamento de Zoología, Facultad de Ciencias Naturales y Oceanográficas, Universidad de Concepción, Concepción, Chile.

*Email: mruizdg@gmail.com

\section{RESUMEN}

Se revisa el estatus taxonómico de Liolaemus islugensis Ortiz \& Marquet, 1987, especie poco conocida del grupo de L. montanus, que fue propuesta como sinónimo menor de L. pantherinus Pellegrin, 1909. La comparación de las descripciones originales de ambas especies y la revisión del material tipo y otros ejemplares, nos permite validar a $L$. islugensis. Se restringe a $L$. pantherinus para Bolivia y Perú, mientras que $L$. islugensis se distribuye en el altiplano de Chile y Bolivia.

Palabras clave: Chile, Liolaemus pantherinus, reptiles.

\section{ABSTRACT}

We review the taxonomic status of Liolaemus islugensis Ortiz \& Marquet, 1987, a scarcely known species belonging to the L. montanus group, that was propose as a junior synonym of $L$. pantherinus Pellegrin, 1909 . The comparison of the original descriptions of both species and the review of the type material and other specimens allow us to validate $L$. islugensis. Liolaemus pantherinus is restricted to Bolivia and Peru, and $L$. islugensis is distributed in highlands of Chile and Bolivia.

Keywords: Chile, Liolaemus pantherinus, reptiles.

Con más de 260 especies de lagartijas (Uetz et al. 2019), el género Liolaemus ha sido considerado uno de los grupos de vertebrados más diversos. En este género se reconocen dos subgéneros: Liolaemus conocido como grupo chileno y Eulaemus conocido como grupo argentino (Laurent 1983, 1985; Etheridge 1995; Schulte et al. 2000).

Dentro del grupo argentino, se encuentra el grupo de Liolaemus montanus (Etheridge 1995) que contiene especies que habitan el desierto y altas altitudes de la cordillera de los Andes en Argentina, Bolivia, Chile y Perú (Donoso-Barros 1966, Cei 1993, Pincheira-Donoso et al. 2008, Aparicio \& Ocampo 2010). Este grupo de lagartijas ha sido escasamente estudiado en Chile (Troncoso-Palacios 2014). Por lo anterior, muchas de las especies del grupo de L. montanus han sido el centro de diversas controversias taxonómicas (Núñez \& Jaksic 1992, Pincheira-Donoso \& Núñez 2002, 2005, Lobo et al. 2010, Troncoso-Palacios 2014, Ruiz de Gamboa et al. 2018).

Liolaemus pantherinus Pellegrin, 1909 fue descrita para el altiplano peruano y boliviano, sin localidad específica. Posteriormente, Donoso-Barros (1966) señala que la tierra típica de esta especie está en las cercanías del Lago Titicaca, sin argumentar al respecto, pero probablemente basado en que todas las otras descripciones de Liolaemus en Pellegrin (1909) tienen localidad tipo en esa zona. Adicionalmente, Donoso- 
Barros (1966) indica que L. pantherinus debe ser incorporada a la fauna chilena para el altiplano de Antofagasta, aunque esto implicaba una ampliación de más de $500 \mathrm{~km}$ en línea recta.

Laurent $(1982,1984)$ sugirió que Liolaemus mocquardi, L. pantherinus y L. pulcher Pellegrin, 1909, corresponden a la hembra, juvenil y macho de la misma especie, pero no realiza una sinonimia. Posteriormente, Laurent (1992) tras estudiar los sintipos de L. pantherinus, indica que estos son "indistinguibles" de L. signifer (Duméril \& Bibron, 1837) pero no realiza una sinonimia explícita. Núñez \& Jaksic (1992) reconocen a $L$. pantherinus como especie distinta de L. signifer y a L. islugensis Ortiz y Marquet, 1987 como nomen dubium. Finalmente, Pincheira-Donoso \& Núñez (2002) proponen a L. islugensis como sinónimo menor de L. pantherinus. Esta sinonimia ha sido reconocida por algunos autores (PincheiraDonoso \& Núñez 2005, Pincheira-Donoso et al. 2008, Vidal et al. 2008), pero otros siguen considerando válidas a ambas especies (Lobo et al. 2010, Abdala \& Quinteros 2014, Ruiz de Gamboa 2016, Uetz et al. 2019).

Dado que la sinonimia de Liolaemus islugensis con $\mathrm{L}$. pantherinus aún genera confusión, en este trabajo se revisa el estatus taxonómico de L. islugensis. Para esto, se revisó la descripción de L. pantherinus, las caracterizaciones posteriores de otros autores (Donoso-Barros 1966, Pincheira-Donoso \& Núñez 2005) y la descripción de L. islugensis. Se revisaron los argumentos brindados por Pincheira-Donoso \& Núñez (2002) para proponer a L. islugensis como sinónimo menor de L. pantherinus. Para actualizar el mapa de distribución se recopilaron las localidades de recolección de ejemplares y citas en la literatura (Donoso-Barros 1966, Ortiz \& Marquet 1987, GBIF 2016, Aguilar-Putriano et al. 2018).

Se examinaron un total de 52 ejemplares de Liolaemus islugensis (Apéndice 1). Esto incluyó al holotipo, alotipo y paratipos de esta especie. Los caracteres para L. pantherinus fueron tomados de (Pellegrin 1909) y se solicitó fotografías de los dos sintipos (MNHNP 05-343 y 05-344) al Museo Nacional de Historia Natural de Paris (MNHNP).

La descripción original de Liolaemus pantherinus (Pellegrin, 1909) es breve y poco detallada. Donoso-Barros (1966) entrega más características de esta especie en base a ejemplares del altiplano de la Región de Antofagasta y es esta última coincidente con la descripción realizada posteriormente por Pincheira-Donoso \& Núñez (2005). Donoso-Barros (1966) revisa dos hembras y un macho, pero no indica voucher ni procedencia del material, mientras que todos los ejemplares examinados por Pincheira-Donoso \& Núñez (2005) son sólo de Chile. Ambas caracterizaciones presentan diferencias en el valor del número de escamas alrededor del medio cuerpo respecto de la descripción original (Tabla 1).

La revisión del material tipo de ambas especies da cuenta de diferencias en el diseño de coloración y el número de escamas al medio del cuerpo. Liolaemus islugensis presenta manchas más pequeñas en la zona dorsal (Fig. 1a), los flancos poseen escamas azules, el vientre presenta escasas marmoraciones (Fig. 1b) y posee un mayor número de escamas al medio cuerpo (rango de $60-78$, promedio $73, \mathrm{~N}=53$ ), a diferencia de $L$. pantherinus que presenta manchas más grandes en la zona dorsal, vientre marmorado (Fig. 2), no presenta escamas azules en los flancos y posee un menor número de escamas al medio cuerpo (rango de 50-52, $\mathrm{N}=2$ ).

Los ejemplares recolectados en Chile no coinciden con la descripción original de Liolaemus pantherinus y deben ser asignados a $L$. islugensis. Cabe destacar que entre estos se encuentran ejemplares determinados como $L$. pantherinus por Donoso-Barros (MZUC-1975-1976 Qda del Inca; MZUC1967-1969, 1971, 1973-1974 Volcán Oyahue; MZUC-31108 Volcán Tatio) para la Región de Antofagasta (Apéndice 1). Estos ejemplares han perdido la notoriedad del diseño dorsal debido a su antigüedad. No obstante, se pueden observar claramente escamas azules en los flancos, el vientre no presenta marmoraciones y el número de escamas alrededor del medio del cuerpo (rango 70-76, promedio $73, \mathrm{~N}=8$ ) se encuentra en el rango de $L$. islugensis.

Pincheira-Donoso \& Núñez (2002) señalan que Liolaemus islugensis y L. pantherinus presentan el mismo tipo de escamas, coloración ventral y diseño dorsal y las diferencias en el número de escamas y tamaño de cola, son justificadas por ser caracteres altamente variables. Cabe destacar que los autores no indican el material examinado. Aquí se presenta evidencia de las diferencias entre ambas especies. Por lo tanto, se sugiere no considerar válida esta sinonimia y se valida a $L$. islugensis como buena especie, la que se distribuye (Fig. 3) en Chile, desde el sur del salar de Surire (Polloquere, Región de Arica y Parinacota) hasta El Tatio (Región de Antofagasta), y en Bolivia (Aguilar-Putriano et al. 2018).

Pellegrin (1909: 325) indica que los sintipos de Liolaemus pantherinus quedaron depositados en el Museo de Historia Natural de París (MNHNP) bajo los códigos 05-344 y 05-345. Sin embargo, el número 05-345 es nombrado nuevamente para L. bolivianus (Pellegrin 1909: 329). La revisión del material depositado en el MNHNP bajo esos códigos evidencia que Pellegrin (1909) erró en este detalle y los números correctos para los sintipos de L. pantherinus son 05-343 y 05-344, mientras que el espécimen 05-345 correspondería a $L$. bolivianus, actualmente sinónimo menor de L. signifer (Duméril \& Bibron, 1837). L. pantherinus estaría restringida al altiplano de Bolivia y Perú, sin embargo, queda aún pendiente la designación de un lectotipo, recopilar mayores antecedentes sobre la localidad tipo precisa y así obtener más ejemplares para detallar su distribución y diagnosis. 


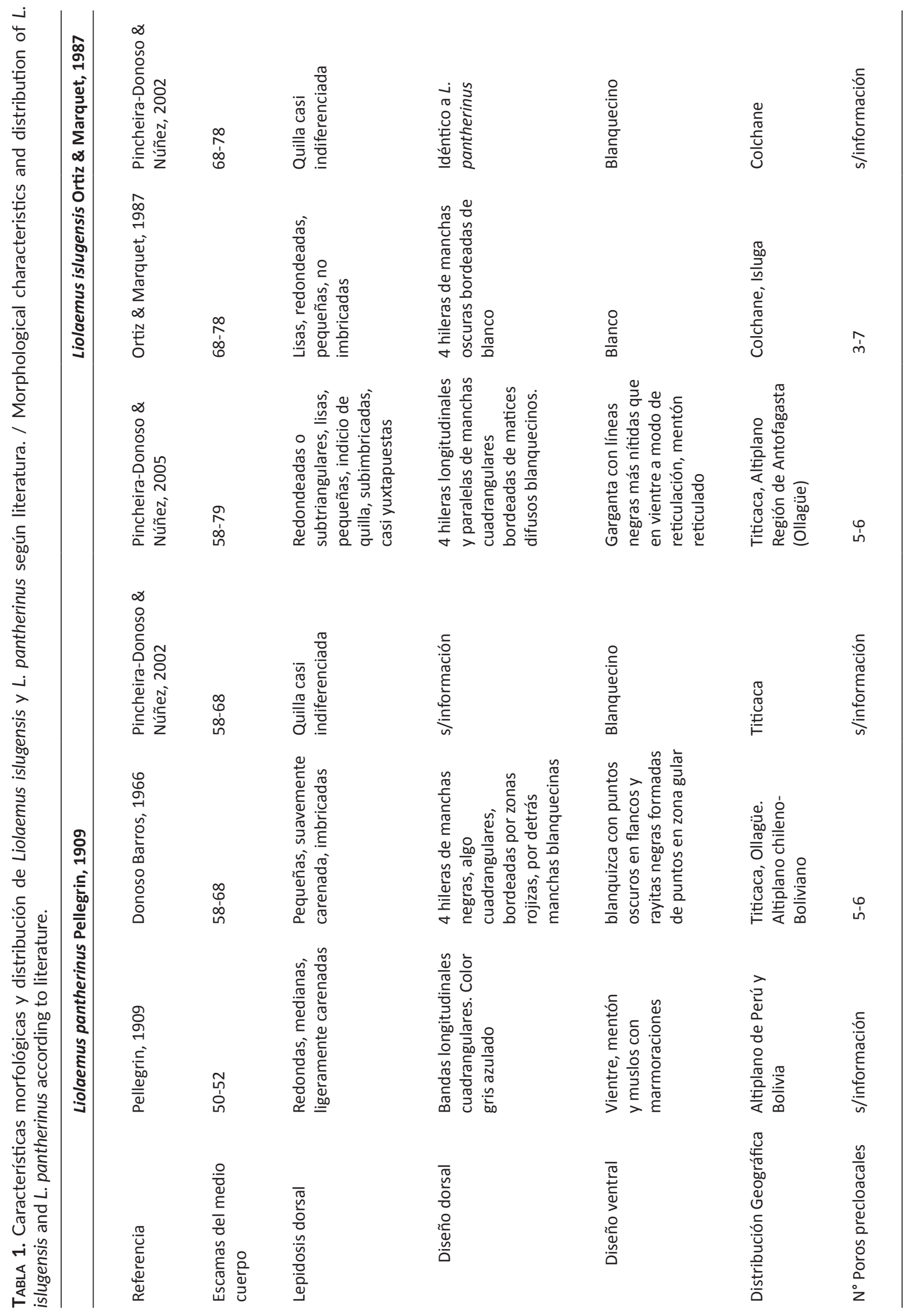




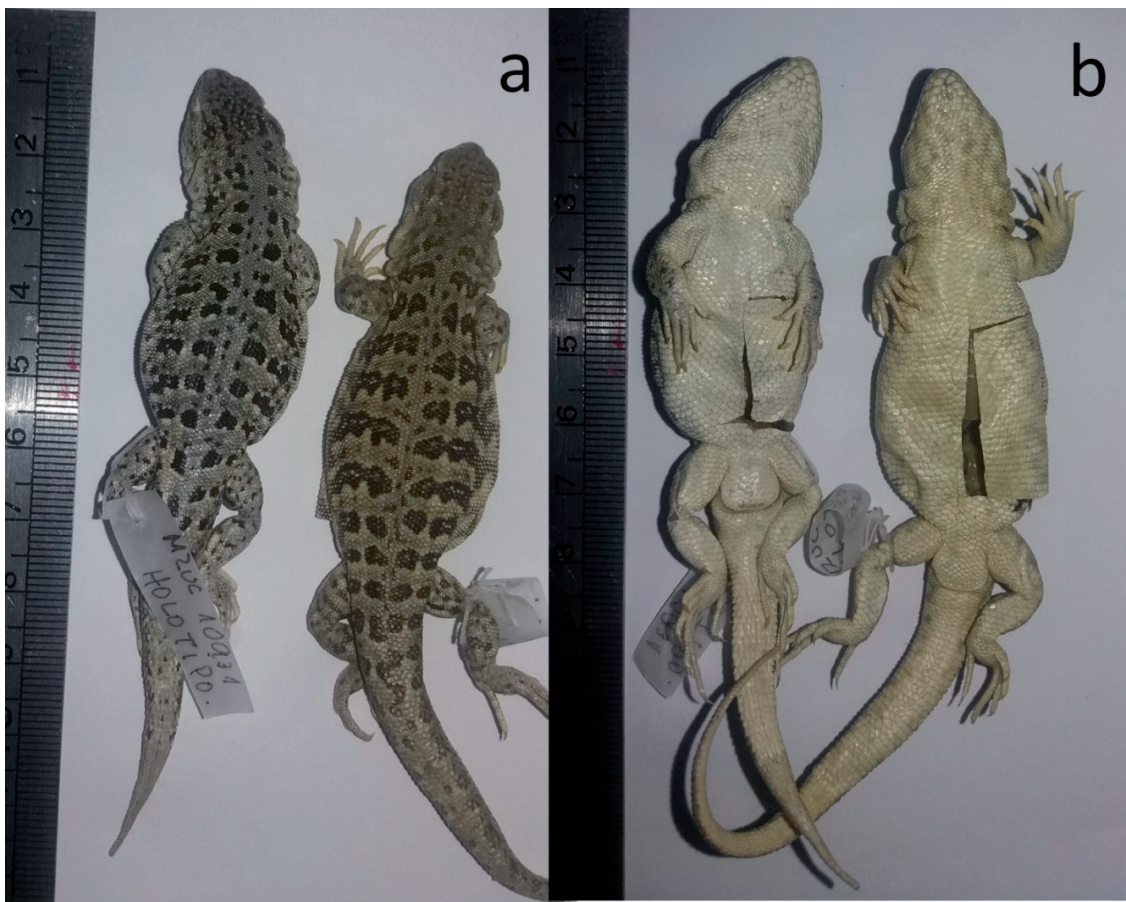

FIGURA 1. a) Vista dorsal y b) vista ventral de Liolaemus islugensis. De izquierda a derecha: Holotipo (MZUC-10931, macho) y Alotipo (MZUC-10932, hembra). / a) Dorsal view and b) ventral view of Liolaemus islugensis. From left to right: Holotype (MZUC-10931, male) and Allotype (MZUC-10932, female).

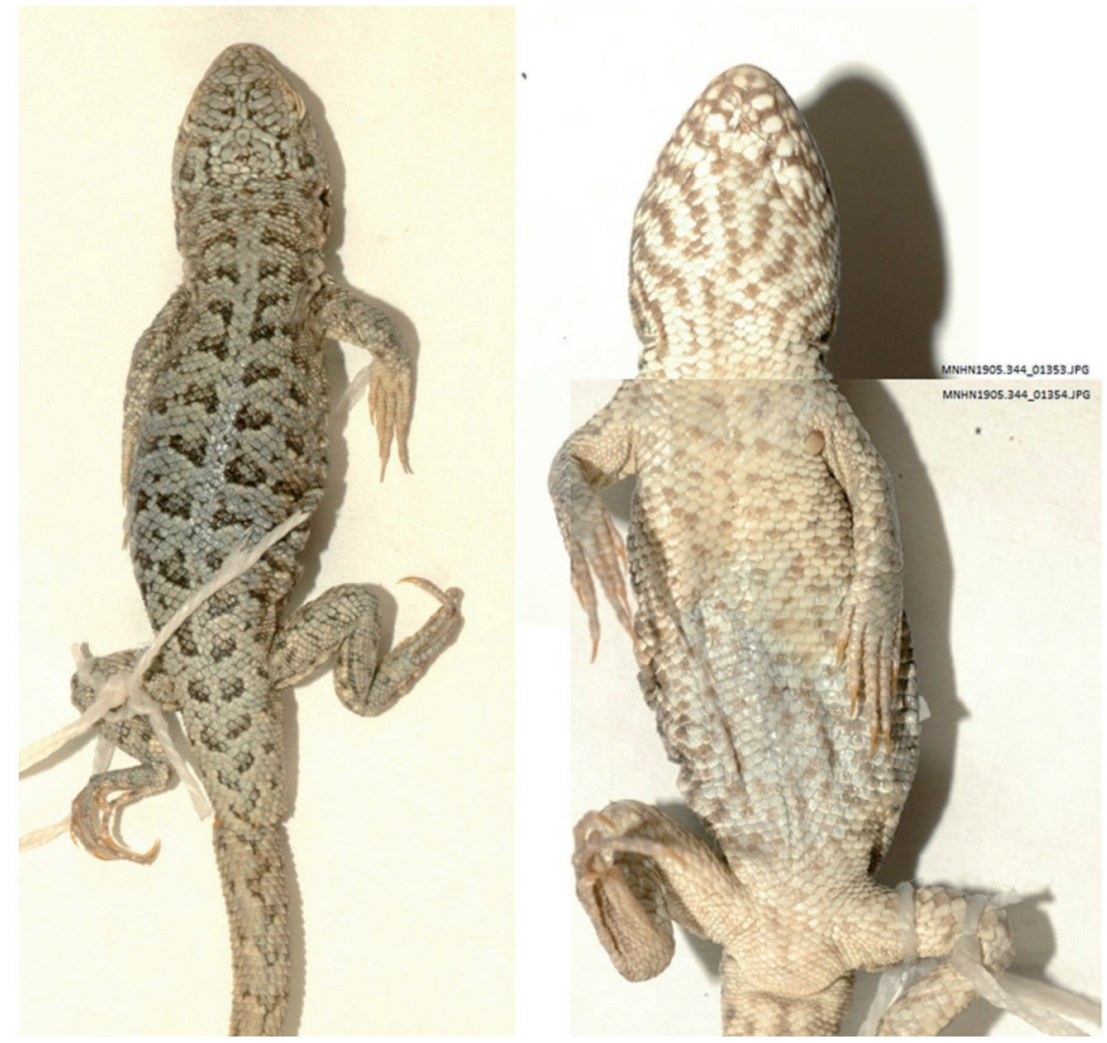

FIGURA 2. Vista dorsal y ventral de Liolaemus pantherinus (sintipo MNHNP 1905-344). / Dorsal and ventral view of Liolaemus pantherinus (syntype MNHNP 1905-344). 


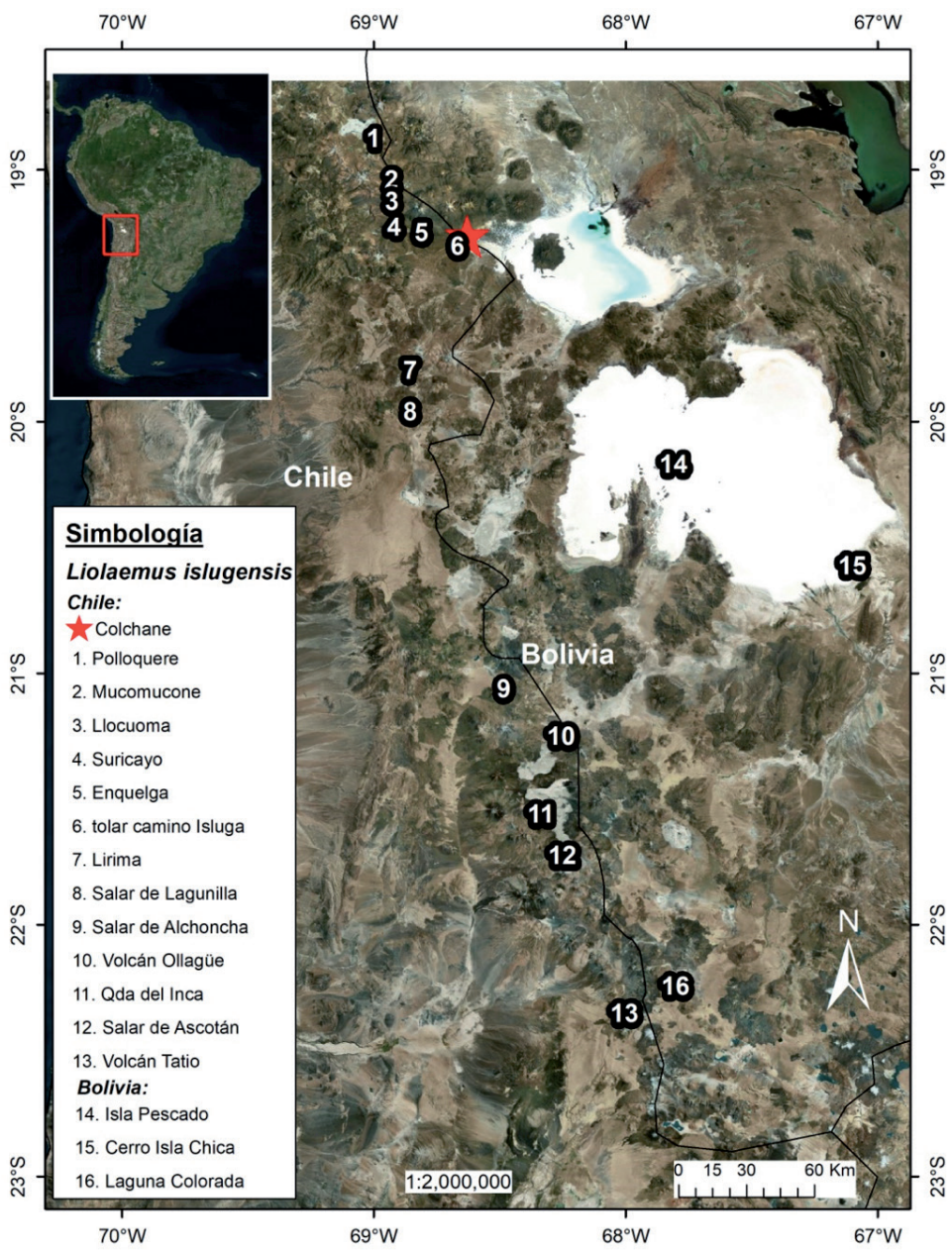

Figura 3. Mapa de distribución actualizado. La estrella roja es la tierra típica de Liolaemus islugensis y los números 7 - 9 corresponden a nuevas localidades de registro (ver apéndice 1). Localidades número 1-6 en Ortiz \& Marquet (1987), 10-11, 13 en Donoso-Barros (1966), 12 en GBIF (2016), 14-16 en Aguilar-Puntriano et al. (2018). / Distribution map update. The star is type locality of Liolaemus islugensis and numbers 7 - 9 correspond to new records (see appendix 1). Localities numbers 1-6 in Ortiz \& Marquet (1987), 10-11, 13 in Donoso-Barros (1966), 12 in GBIF (2018), 14-16 en Aguilar-Puntriano et al. (2018).

\section{AGRADECIMIENTOS}

Se agradece al Museo Nacional de Historia Natural de París por el envío de fotografías digitales, en especial a Dr. Annemarie Ohler, Curador de Lepidosauria y Amphibia en Muséum national d'Histoire naturelle. También agradecemos el valioso aporte de los revisores, quienes ayudaron a mejorar sustancialmente este artículo.

\section{REFERENCIAS}

Abdala, C., Quinteros, A. 2014. Los últimos 30 años de estudios de la familia de lagartijas más diversa de Argentina. Actualización taxonómica y sistemática de Liolaemidae. Cuadernos de Herpetología 28(2): 55-82.
Aparicio, J., Ocampo, M. 2010. Liolaemus grupo montanus Etheridge, 1995 (Iguania - Liolaemidae). Novedad zoogeográfica. Cuadernos de Herpetología 24(2): 133135.

Aguilar-Puntriano, C., Avila, L., De la Riva, I., Johnson, L., Morando, M., Troncoso-Palacios, J., Wood, P. Jr., Sites, J. Jr. 2018. The shadow of the past: Convergence of young and old South American desert lizards as measured by head shape traits. Ecology and Evolution. 8: 11399-11409.

Cei, J.M. 1993. Reptiles del noroeste, nordeste y este de la Argentina. Herpetofauna de las selvas subtropicales, Puna y Pampas. Museo Regionale di Scienze Naturali di Torino, Monografie 14. 949 pp.

Donoso-Barros, R. 1966. Reptiles de Chile. Ediciones de la Universidad de Chile, Santiago de Chile. 458 pp.

Etheridge, R. 1995. Redescription of Ctenoblepharys adspersa 
Tschudi, 1845, and the taxonomy of Liolaeminae (Reptilia: Squamata: Tropiduridae) American Museum Novitates 3142: 1-34.

GBIF.org (28 Diciembre 2016) GBIF Occurrence Download. URL: https://www.gbif.org/occurrence/ download/0045186-160910150852091 (Acceso Mayo 11, 2018)

Laurent, R. 1982. Las especies y "variedades" de Liolaemus descritas por J. Koslowsky (Sauria Iguanidae). Neotropica 28: 87-96.

Laurent, R. 1983. Contribución al conocimiento de la estructura taxonómica del género Liolaemus Wiegmann (Iguanidae). Boletín de la Asociación Herpetológica Argentina 1: 16-18.

Laurent, R. 1984. Fenogramas de algunas especies representativas del género Liolaemus y generos vecinos (iguanidae, reptilia). Acta zoológica lilloana 38(1): 5-17.

Laurent, R. 1985. Segunda contribución al conocimiento de la estructura taxonómica del género Liolaemus Wiegmann (Iguanidae). Cuadernos de Herpetología 1: 1-37.

Laurent, R. 1992. On some overlooked species of the genus Liolaemus Wiegmann (Reptilia- Tropiduridae) from Peru. Breviora 494: 1-33.

Lobo, F., Espinoza, R.E., Quinteros, A.S. 2010. A critical review and systematic discussion of recent classification proposals for liolaemid lizards. Zootaxa 2549: 1-30.

Núñez, H., Jaksic, F. 1992. Lista comentada de los reptiles terrestres de Chile Continental. Boletín del Museo Nacional de Historia Natural, Chile 43: 63-91.

Ortiz, J., Marquet, P. 1987. Una nueva especie de lagarto altoandino: Liolaemus islugensis (Reptilia-Iguanidae) Gayana Zoología 51(1-4): 59-63.

Pellegrin, J. 1909. Description de cinq lézards nouveaux des hauts-plateaux du Pérou et de la Bolivie, appartenant au genre Liolaemus. Bulletin du Muséum National d'Histoire Naturelle Paris 6: 324-329.
Pincheira-Donoso, D., Núñez, H. 2002. Situación taxonómica de Liolaemus ornatus Koslowsky, 1898 y Liolaemus pantherinus Pellegrin, 1909 (Sauria, Tropiduridae, Liolaeminae). Noticiario mensual del Museo de Historia Natural de Chile 350: 38-42.

Pincheira-Donoso, D., Núñez, N. 2005. Las especies Chilenas del género Liolaemus Wiegmann, 1834 (Iguania: Tropiduridae: Lioleminae) Taxonomía, Sistemática y Evolución. Publicación Ocasional del Museo de Historia Natural Chile 59: 7-486.

Pincheira-Donoso, D., Scolaro, J.A., Sura, P. 2008. A monographic catalogue on the systematics and phylogeny of the South American iguanian lizard family Liolaemidae (Squamata, Iguania). Zootaxa 1800: 1-85.

Ruiz de Gamboa, M. 2016. Lista actualizada de los reptiles de Chile. Boletín Chileno de Herpetología 3: 7-12.

Ruiz de Gamboa M., Correa, C., Marambio-Alfaro, Y., Riveros-Riffo E., Ortiz, J.C. 2018. Molecular evidence for conspecificity of two desert Liolaemus lizards (Iguania: Liolaemidae). Zootaxa 4438(2): 283-298.

Schulte, J.A.II., Macey, J.R., Espinoza, R.E., Larson, A. 2000. Phylogenetic relationships in the iguanid lizard genus Liolaemus: multiple origins of viviparous reproduction and evidence for recurring Andean vicariance and dispersal. Biological Journal of the Linnean Society 69: 75-102. https://doi.org/10.1006/bijl.1999.0346

Troncoso-Palacios, J. 2014. Revisión del estatus taxonómico de Liolaemus filiorum Pincheira-Donoso y Ramírez, 2005 (Iguania: Liolaemidae). Cuadernos de Herpetología 28: 111-117.

Uetz, P., Freed, P., Hošek, J. (Eds) 2019. The Reptile Database. URL: http://www.reptile-database.org. Accessed October 2, 2019.

Vidal, M., Iturra-Cid, M., J.C., Ortiz. 2008. Clasificación de anfibios y reptiles. En: Vidal, M., Labra, A. (Ed) Herpetología de Chile: 79-106. Science Verlag, Santiago, Chile.

APÉNDICE 1. Material examinado. / Material examined.

Liolaemus islugensis

Chile: Región de Arica y Parinacota: 18321 Polloquere; Región de Tarapacá: MZUC-10931 Colchane fte a Santuario, Holotipo; MZUC-10932 Colchane, Alotipo. Paratipos: MZUC-10934, MZUC-18275 tolar camino Isluga; MZUC-10936-10937, MZUC-10939-10947 Colchane; MZUC-10949-10950 Adesmia; MZUC-10950; MZUC-18317-18318 Mucomucone; MZUC18319-18320 Llocuoma; MZUC-18333 Enquelga; MZUC18334-18335 Suricayo; MZUC-33493 Salar de Alconcha; MUAP-127 - 144 Salar de Lagunilla; MUAP-145 Lirima. Región de Antofagasta: MZUC-1975-1976 Qda del Inca; MZUC-1967-
1969, 1971, 1973-1974 Volcán Oyahue; MZUC-31108 Volcán Tatio (como L. pantherinus).

Liolaemus pantherinus

Sintipos MNHNP 1905-343 y 1905-344 Sin localidad, señalada por Pellegrin (1909) para el altiplano peruano y boliviano.

Liolaemus signifer

MNHNP 1905-345 Sin localidad, señalada por Pellegrin (1909) para el altiplano peruano y boliviano (como L. bolivianus). 